\title{
The Effect of Velocity on Load Range During Isokinetic Hip Abduction and Adduction Exercise
}

\section{ABSTRACT}

The purpose of this study was to quantify the components of acceleration, load range and deceleration through a velocity spectrum during concentric hip abduction and adduction isokinetic exercise, and to investigate the effect of load range on peak torque and work done. Sixteen male healthy subjects performed three maximal concentric reciprocal hip abduction and adduction gravity corrected repetitions in a fixed order at $60,120,180,240,300,360$ and $420^{\circ} \cdot \mathrm{s}^{-1}$, with a 30 second rest between velocities. Hip abduction and adduction results revealed that load range significantly decreased while acceleration and deceleration ROM significantly increased $(p<0.05)$ with each increase in velocity. When the total peak torque data was corrected for load range there was a significant decrease $(p<0.05)$ in peak torque at velocities of $300^{\circ} \cdot \mathrm{s}^{-1}$ and above, for both hip abduction and adduction. Load range correction also resulted in a significant decrease $(p<0.05)$ in work done at velocities of $120^{\circ} \cdot \mathrm{s}^{-1}$ and above, for both hip abduction and adduction. The results demonstrate an inverse relationship between isokinetic velocity and load range during concentric hip abduction and adduction, and suggest a need for the clinician to carefully consider velocity selection when performing exercise on an isokinetic device.

\section{Key Words}


Isokinetic Exercise; Acceleration; Deceleration; Peak Torque; Work Done.

\section{INTRODUCTION}

The isokinetic dynamometer has commonly been used for rehabilitation or training purposes $[4,1 \underline{6} 5,1 \underline{7} 6,2 \underline{6} 5,2 \underline{7} 6, \underline{31} 30]$. As the isokinetic dynamometer only offers resistance once the pre-set velocity is attained, any strength gains achieved from isokinetic exercise may be proportional to the total amount of range of motion (ROM) actually sustained at the pre-set isokinetic velocity [8]. It is therefore of great interest to investigate what percentage of the ROM of a concentric actionisokinetic contraction-is actually spent at the pre-selected velocity, over a velocity spectrum.

A concentric action contractionperformed on an isokinetic device involves three main components: acceleration, sustained velocity, and deceleration [8, 298, 3ㅁ5]. The acceleration component has been defined as the individual's ability to "catch" the dynamometer [10, 3029]. The "catch" phase is completed once the individual attains the pre-set velocity, and the resistance is met, which then prevents any further acceleration $[10,3029]$. The sustained velocity component of the repetition has also been termed load range $[8,1 \underline{3} z, 2 \underline{2} 1]$. To be more precise the concept of load range has been described as external machine resistance encountered through a pre-set sustained velocity within a defined range of motion (ROM) [7]. The final component, mechanical deceleration, offers resistance while the isokinetic dynamometer decreases speed at the end of the defined ROM. However, Brown et al. [8] has argued that this phase is neither directly governed by the tester nor quantifiable as 
torque produced under controlled isokinetic conditions, and therefore ceases to be isokinetic [8].

Earlier research has shown that torque patterns are significantly affected when the load range phase of the motion is taken into consideration $[3,8,210]$. In short, this means that actual torque may differ by a large magnitude if evaluated outside of the load range [1르]. Kurdak et al. [2ㄹ1] found a significant decrease when comparing load range peak torque to total peak torque at speeds above $270^{\circ} \cdot \mathrm{s}^{-1}$ for knee extension and above $300^{\circ} \cdot \mathrm{s}^{-1}$ for knee flexion. The authors also found a significant decrease when comparing load range work and total work at speeds above $90^{\circ} \cdot \mathrm{s}^{-1}$ for both knee extension and knee flexion. These results highlight the importance of correcting the data for load range as it is apparent that large errors can occur if this process is not undertaken.

Increased angular velocity results in a reduction in load range, thus data from the measurements that were performed at higher angular velocities may not actually reflect load range values [221]. This is in agreement with the classic force - velocity curve, which explains the relationship between skeletal muscle contraction velocity and torque production [3ㅁ7]: as velocity increases, torque decreasesforce increases, velocity decreases [3]. Therefore extra caution is required to make correct interpretation of isokinetic results [3].

Load range has been investigated previously, however, only during unilateral knee flexion/extension [8, $\underline{3029}, 3 \underline{7} 5, \underline{4139}$ ], bilateral knee flexion/extension [3ㅁ4] and shoulder external/internal rotation [7]. Each study found an inverse relationship 
between load range and velocity, yet the primary focus of these studies was load range, apart from Brown et al. [8] who also considered the impact of the acceleration and deceleration components. Therefore, quantification of each component may lead to a more complete understanding of load range magnitude and position within the exercised ROM. This information may better equip the clinician in more accurate velocity prescription during isokinetic exercise.

Recently the investigation into the musculature around the hip has become of interest, especially in regards to patients with a history of functional ankle instability (FAI) [1 $\underline{5} 4]$. It has been suggested that patients with a history of FAI may have a weakness in muscles surrounding the hip, primarily the gluteus medius, which results in a more adducted foot placement during the gait cycle [143, 287]. This adducted foot position results in an increased chance of the individual contacting the floor with the lateral aspect of the foot, which could potentially lead to an increased chance of 'rolling over' on the ankle and sustaining a lateral ankle sprain [1ㄴ3]. O'Dwyer et al. [2즈] stated that dysfunction of the gluteus medius is commonly implicated in lower limb pathologies. It has been stated that the gluteus medius muscle should be evaluated in healthy participants, to try and identify individuals with a possible predisposition to ankle sprains [1].

The primary aim of this study was therefore to quantify the components of load range, acceleration, and deceleration through a velocity spectrum during concentric hip abduction and adduction isokinetic exercise. The secondary aim of this study was to investigate the effect of load range on peak torque and work done. 


\section{METHOD}

\section{Subjects}

Sixteen male healthy subjects (age $=21.2 \pm 2.2$ years, height $=182.7 \pm 4.8 \mathrm{~cm}$, and mass $=77.4 \pm 5.1 \mathrm{~kg}$ ) volunteered to participate in the study. Institutional ethical approval was granted for this study, and all subjects provided written informed consent before participation. This study was conducted in accordance with recognised ethical standards and national/international laws [1ㅁ7]. Inclusion criteria included males, aged 18-25 years, who participated in regular exercise (low to moderate aerobic exercise two to three times per week), and who were right leg dominant. The dominant leg was defined as the preferred kicking leg. Subjects were excluded from the study if they had a cold or flu in the past two weeks, if they suffered from any musculoskeletal injuries, ankle, knee or hip injuries, or fractures to the lower limbs in the last year. Subjects were asked to refrain from any vigorous exercise 48 hours prior to testing.

\section{Experimental Design}

\section{Physiological Procedures}

Subject's age, mass and height was recorded. A warm-up was accomplished by a five minute cycle on a Monark cycle ergometer (Monark, Varberg, Sweden) at 50rpm with a resistance of 50 Watts. Testing was performed on the Biodex System 2 Isokinetic Dynamometer (Biodex Medical Systems, Shirley, New York). The system reliability of the Biodex dynamometer has been shown to be high, with Intraclass 
Correlation Coefficient's $\left(\mathrm{ICC}_{(2,1)}\right)$ ranging from $0.92-0.98$ for peak torque and 0.880.97 for total work [5]. Taylor et al. [375] also demonstrated the mechanical validity of the Biodex isokinetic dynamometer in relation to human torque, joint position and limb velocity.

The Biodex was set up according to the Biodex System 2 Manual, and was calibrated according to manufacturer's specifications prior to testing. The cushion control was set to zero, to allow the subject the greatest availability of velocity attainment prior to deceleration [8, 375]. All subjects completed a practice session on the isokinetic dynamometer a week prior to the main testing procedure.

Subjects were side lying, facing away from the dynamometer power head, with the right hip superior. The right knee was extended, and the left knee was flexed to $90^{\circ}$. The axis of the dynamometer was aligned superior and medial to the greater trochanter of the right leg. The subject's right leg was attached to the Biodex hip attachment, superior to the lateral knee joint line. The subject's ROM was set to 0$45^{\circ}$ of abduction. The ROM was based on the average limitations of hip motion in healthy individuals $[11,243,3 \underline{4} 3]$. The pelvis was stabilised using the straps of the dynamometer and the subject's top hand grasped the border of the chair. The side lying single chair hip abduction/adduction protocol was chosen, as opposed to a standing hip abduction/adduction protocol, due to the chair and straps of the isokinetic dynamometer being able to control for torso and pelvis movement. It may be argued that standing hip abduction/adduction has more relevance to walking, however, the design of the system 2 dynamometer does not allow for support of the torso and pelvis during the standing protocol [1ㅁㄴ]. 
Warm-up on the isokinetic device consisted of three submaximal reciprocal concentric abduction and adduction repetitions with increasing intensity (i.e. first repetition at $25 \%$ perceived effort, second repetition at $50 \%$ perceived effort, and third repetition at $75 \%$ perceived effort) [8], at $60^{\circ} \cdot \mathrm{s}^{-1}$ through $420^{\circ} \cdot \mathrm{s}^{-1}[5,3 \underline{8} 6]$. In addition the subject completed two maximal intensity repetitions at each speed [8, $1 \underline{3} 2]$.

Testing began from a dead stop [8] with the subject's leg at $0^{\circ}$ of abduction and consisted of three maximal concentric reciprocal hip abduction and adduction gravity corrected repetitions in a fixed order at $60,120,180,240,300,360$ and $420^{\circ} \cdot \mathrm{s}^{-1}$, with a 30 second rest between velocities [3ㅇ6]. Each subject was encouraged to contact the mechanical end stops during both abduction and adduction movements. The same verbal encouragement was given to each subject throughout the test to motivate them to develop maximal torque during each repetition [2ㄷ4] but no visual feedback of torque generation was provided.

\section{Mechanical Procedures}

To determine the mechanical deceleration effect of the dynamometer, a test similar to that presented by Brown et al. [8] was performed. The Biodex calibration-weighted lever arm was dropped through the same range of motion (ROM) as performed by the human subjects $\left(0-45^{\circ}\right)$. Nine repetitions were recorded at velocities of 60,120 , 180 , and $240^{\circ} \cdot \mathrm{s}^{-1}$. These values were used for comparison with the deceleration values recorded by human subjects (ie, physiological deceleration) [8]. Mechanical procedures could not be performed at the last three test velocities due to the inability 
of gravity to accelerate the weighted lever to speeds of 300,360 and $420^{\circ} \cdot \mathrm{s}^{-1}$ within the specified ROM.

\section{Data Analysis}

Data was collected via the Biodex Advantage Software (version 4.5, Biodex Medical Systems, Shirley, New York), which allowed the separation of each contraction into its component parts for individual analysis. The same definitions as stated by Brown et al. [8] were used; the range of motion prior to velocity attainment was termed acceleration, while ROM after load range was termed deceleration (Figures 1 and 2). Load range was determined for hip abduction and adduction by subtracting the sum of acceleration ROM and deceleration ROM from the total test ROM using the available cursors on the screen [2, 4, 6, 21ㅂ, 4038]. Taylor et al [3프] stated that velocity overshoot was measured at $3.5 \%$ on the Biodex dynamometer, this is not reflected in the velocity tracings but was included in the load range component. Brown et al. [8] recommended using $100 \%$ of the pre-selected velocity because there is no machine-offered resistance below full velocity attainment $[7,2 \underline{9} 8, \underline{30} 29,3 \underline{2} 7$, $3 \underline{6} 4,3 \underline{75}, \underline{4038}]$.

Following the determination of load range ROM, the total peak torque, load range peak torque, joint angle at load range peak torque, total work and load range work were calculated for both hip abduction and hip adduction across all velocities. Total peak torque was determined by locating the highest point of the curve. The load range peak torque was determined by locating the highest point of the curve within the load range ROM. The joint angle at which this load range peak torque occurred 
was then recorded. Total work done was determined by calculating the area under the curve. The load range work done was determined by calculating the area under the curve within the load range ROM. All torque data was normalised with respect to the subject's body weight [22ㅍ].

\section{Statistical Analysis}

Using SPSS (version 19) a $7 \times 2$ (speed $\left[60,120,180,240,300,360\right.$ and $\left.420^{\circ} \cdot \mathrm{s}^{-1}\right] \mathrm{x}$ muscle action [hip abduction and hip adduction]) mixed factorial repeated measures analysis of variance (ANOVA) was performed for the acceleration, load range and $\underline{\text { deceleration data to identify if a significant difference was present across velocities }}$ $(p<0.05)$. The results were studied for two way interactions and then main effects.

A $7 \times 2 \times 2$ (speed $\left[60,120,180,240,300,360\right.$ and $\left.420^{\circ} \cdot \mathrm{s}^{-1}\right] \times$ condition [total $\underline{\text { values and load range values] x muscle action [hip abduction and hip adduction]) }}$ $\underline{\text { mixed factorial repeated measures ANOVA was performed for peak torque and work }}$ data $(p<0.05)$. The results were studied for three way interactions, then two way interactions and then main effects.

To determine the difference between mechanical and physiological deceleration across velocities a $4 \times 2\left(\right.$ speed $\left[60,120,180\right.$, and $\left.240^{\circ} \cdot \mathrm{s}^{-1}\right] \times$ condition [mechanical or physiological deceleration]) mixed factorial repeated measures ANOVA was performed $(p<0.05)$. Results were studied for two way interactions and then main effects. 
Using SPSS (version 19) sixteen repeated measures analysis of variance (ANOVA) were used to analyse the mean value of each action (hip abduction and hip adduction) by each dependent variable (acceleration, load range, deceleration, total peak torque, load range peak torque, joint angle at load range peak torque, total work, and load range work) between velocities $(p<0.05)$.

A two-way (speed $\left[60,120,180\right.$, and $\left.240^{\circ} \cdot s^{-1}\right] \times$ condition [mechanical of physiological deceleration]) mixed factorial repeated measures analysis of variance (ANOVA) was performed to determine the difference between mechanical and physiological deceleration across velocities $(p<0.05)$.

Four two-way (speed $\left[60,120,180\right.$, and $\left.240^{\circ} \cdot s^{-1}\right] \times$ condition [total values vs. load range values]) mixed factorial repeated measures analysis of variance were performed to determine the difference between total peak torque and load range peak torque, and total work and load range work during both hip abduction and hip adduction across velocities $(p<0.05)$.

\section{RESULTS}

Analysis of variance-The $7 \times 2$ (speed $\times$ muscle action) mixed factorial repeated measures ANOVA results revealed that load range significantly decreased while acceleration and deceleration ROM significantly increased with each increase in velocity, for both hip abduction and hip adduction (Table 1). There was no significant difference found between abduction and adduction results. The amount of ROM spent in load range significantly decreased from $41.8^{\circ}$ to $21.7^{\circ}$ for abduction, and 
from $42.2^{\circ}$ to $22.2^{\circ}$ for adduction, at 60 through $360^{\circ} \cdot \mathrm{s}^{-1}$. The amount of ROM spent in acceleration significantly increased from $1.1^{\circ}$ to $11.1^{\circ}$ for abduction, and from $0.9^{\circ}$ to $10.7^{\circ}$ for adduction, at 60 through $360^{\circ} \cdot \mathrm{s}^{-1}$. The amount of ROM spent in deceleration significantly increased from $2.1^{\circ}$ to $12.2^{\circ}$ for abduction, and from $1.9^{\circ}$ to $12.1^{\circ}$ for adduction, at 60 through $360^{\circ} \cdot \mathrm{s}^{-1}$. Observing the results as a percentage of the total test ROM the abduction load range (Figure 3) significantly decreased from $92.9 \%$ to $48.2 \%$, and adduction load range (Figure 4 ) significantly decreased from $93.8 \%$ to $49.3 \%$, at 60 through $360^{\circ} \cdot \mathrm{s}^{-1}$ respectively. No subjects were able to achieve hip abduction or adduction at $420^{\circ} \cdot \mathrm{s}^{-1}$.

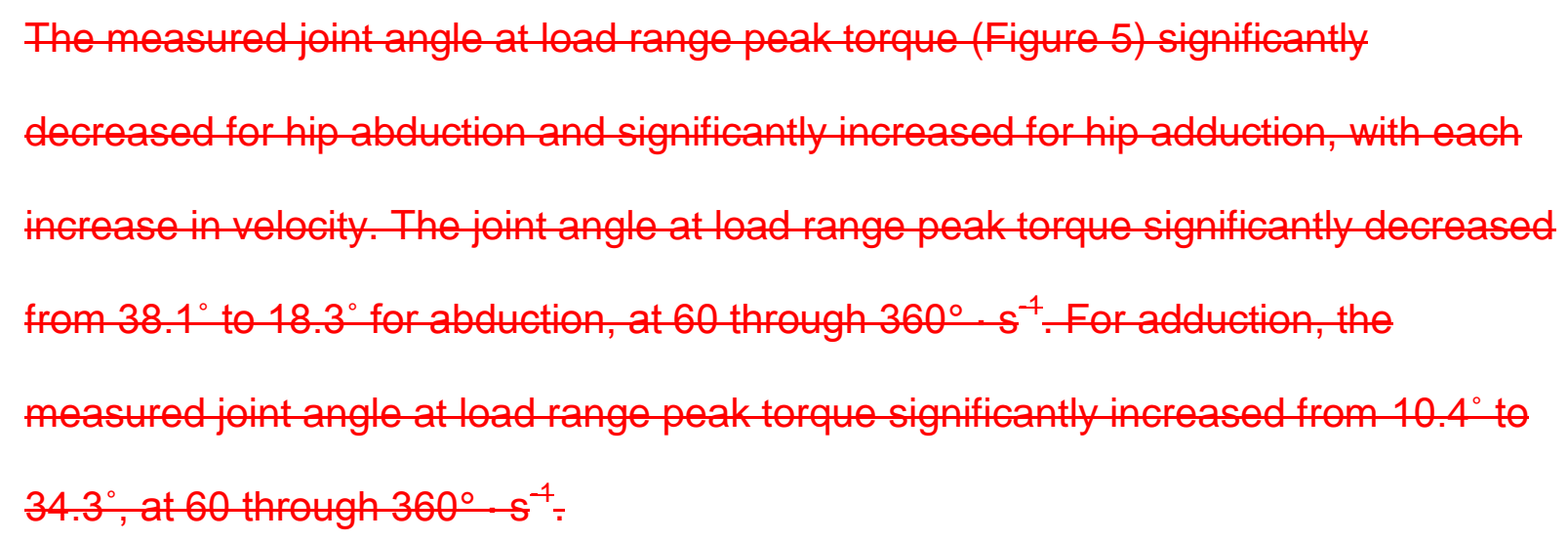

The $7 \times 2 \times 2$ (speed $\times$ condition $\times$ muscle action) mixed factorial repeated measures

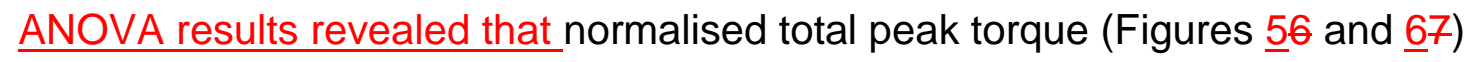
values significantly decreased with each increase in velocity for both hip abduction and hip adduction. There was no significant difference found between hip abduction and adduction results. The normalised total peak torque values significantly decreased from $1.2 \mathrm{Nm} \cdot \mathrm{Kg}^{-1}$ to $0.4 \mathrm{Nm} \cdot \mathrm{Kg}^{-1}$ for abduction, and from $1.0 \mathrm{Nm} \cdot \mathrm{Kg}^{-1}$ to $0.37 \mathrm{Nm} \cdot \mathrm{Kg}^{-1}$ for adduction, at 60 through $360^{\circ} \cdot \mathrm{s}^{-1}$. 
Normalised load range peak torque (Figures $\underline{5} 6$ and $\underline{6} 7$ ) values significantly decreased with each increase in velocity for both hip abduction and hip adduction. There was no significant difference found between hip abduction and adduction $\underline{\text { results. }}$ The normalised load range peak torque values significantly decreased from $1.2 \mathrm{Nm} \cdot \mathrm{Kg}^{-1}$ to $0.2 \mathrm{Nm} \cdot \mathrm{Kg}^{-1}$ for abduction, and from $1.0 \mathrm{Nm} \cdot \mathrm{Kg}^{-1}$ to $0.1 \mathrm{Nm} \cdot \mathrm{Kg}^{-1}$ for adduction, at 60 through $360^{\circ} \cdot \mathrm{s}^{-1}$. The-two-way analysis of variance $7 \times 2 \times 2$ mixed factorial repeated measures ANOVA results also showed a significant difference between normalised total peak torque and load range peak torque from speeds of $300^{\circ} \cdot \mathrm{s}^{-1}$ and above for both hip abduction and hip adduction.

\section{The $7 \times 2 \times 2$ (speed $\times$ condition $\times$ muscle action) mixed factorial repeated measures} ANOVA results revealed that normalised total work (Figures $\underline{78}$ and $\underline{8} 9$ ) values significantly decreased with each increase in velocity for both hip abduction and hip adduction. There was no significant difference found between hip abduction and adduction results. The normalised total work values significantly decreased from 0.85 $\mathrm{Nm} \cdot \mathrm{Kg}^{-1}$ to $0.5 \mathrm{Nm} \cdot \mathrm{Kg}^{-1}$ for abduction, and from $0.84 \mathrm{Nm} \cdot \mathrm{Kg}^{-1}$ to $0.49 \mathrm{Nm} \cdot \mathrm{Kg}^{-1}$ for adduction, at 60 through $360^{\circ} \cdot \mathrm{s}^{-1}$.

Normalised load range work (Figures $\underline{7} 8$ and $\underline{8} 9$ ) values significantly decreased with each increase in velocity for both hip abduction and hip adduction. There was no significant difference found between hip abduction and adduction results. The normalised load range work values significantly decreased from $0.82 \mathrm{Nm} \cdot \mathrm{Kg}^{-1}$ to 0.11 $\mathrm{Nm} \cdot \mathrm{Kg}^{-1}$ for abduction, and from $0.79 \mathrm{Nm} \cdot \mathrm{Kg}^{-1}$ to $0.09 \mathrm{Nm} \cdot \mathrm{Kg}^{-1}$ for adduction, at 60 through $360^{\circ} \cdot \mathrm{s}^{-1}$. The two-way analysis of variance $7 \times 2 \times 2$ mixed factorial repeated measures ANOVA results also showed a significant difference between 
normalised total work and load range work from speeds of $120^{\circ} \cdot \mathrm{s}^{-1}$ and above for both hip abduction and hip adduction.

The two-way analysis of variance $4 \times 2$ (speed $x$ condition) mixed factorial repeated measures ANOVA results revealed that mechanical deceleration ROM significantly increased with each increase in velocity (Table 2), but was not significantly different from the physiological ROM. The mechanical deceleration ROM significantly increased from $1.8^{\circ}$ to $7.9^{\circ}$, at 60 through $240^{\circ} \cdot \mathrm{s}^{-1}$. As previously stated the mechanical deceleration procedure could not be performed at the last three test velocities due to the inability of gravity to accelerate the weighted lever to speeds of 300,360 and $420^{\circ} \cdot \mathrm{s}^{-1}$ within the specified ROM.

It is important to reiterate the point that no subject managed to attain the speed of $\underline{420^{\circ} \cdot \mathrm{s}^{-1} \text {. As load range only occurs once the subject reaches the pre-selected }}$ velocity and the resistance is encountered, no load range data could be produced for this speed. However, it is important to mention that even though load range was not achieved, the Biodex still reported data. This non-corrected data has not been presented in any of the Tables or Figures in this paper, but we thought it was important to mention the data, as it highlights the importance of load range correction.

The normalised total peak torque values produced by the Biodex for the speed of $\underline{420^{\circ} \cdot \mathrm{s}^{-1} \text { were } 0.31 \mathrm{Nm} \cdot \mathrm{Kg}^{-1} \text { for abduction, and } 0.28 \mathrm{Nm} \cdot \mathrm{Kg}^{-1} \text { for adduction. These }}$ non-load range corrected values are higher than the load range corrected values for $\underline{\text { the slower speed of } 360^{\circ} \cdot \mathrm{s}^{-1} \text {, for both hip abduction and adduction. This indicates }}$ 
that without load range correction, there are large errors in the values produced. This error is even more present when observing the normalised total work data produced by the Biodex at $420^{\circ} \cdot \mathrm{s}^{-1}$. Normalised total work for abduction was $0.45 \mathrm{Nm} \cdot \mathrm{Kg}^{-1}$, and for adduction was $0.43 \mathrm{Nm} \cdot \mathrm{Kg}^{-1}$. These non-load range corrected values are much higher than the load range corrected values for the slower speeds of 240,300 and $360^{\circ} \cdot \mathrm{s}^{-1}$, for both hip abduction and adduction. This again indicates that without load range correction, there are large errors present in the data.

With further observation of the traces, a large aspect of the non-corrected peak torque and work values were due to impact artifact. Impact artifact occurs when the dynamometer begins to slow the lever arm in preparation for stopping, and ultimately results in a large isometric spike at the end of the repetition due to the lever arm impacting the mechanical end stop [3]. In several traces, many of the total peak torque values were obtained solely from impact artifact, and a large proportion of the total work values were again due to impact artifact. These results further emphasise the importance of load range correction, as otherwise peak torque and work data is fairly meaningless or largely impact artifact. In addition to this, as no subject could reach the speed of $420^{\circ} \cdot \mathrm{s}^{-1}$, it highlights the importance for future investigators to trial their selected isokinetic velocities during practice sessions to confirm that subjects can attain them.

\section{DISCUSSION}

This study aimed to quantify the components of load range, acceleration, and deceleration through a velocity spectrum during concentric hip abduction and 
adduction isokinetic exercise. The secondary aim of the study was to investigate the effect of load range on peak torque and work done. It is apparent from the results that load range significantly decreased while acceleration and deceleration ROM significantly increased with each increase in velocity, for both hip abduction and hip adduction. When the total peak torque data was corrected for load range there was a significant decrease in peak torque at velocities of $300^{\circ} \cdot \mathrm{s}^{-1}$ and above for both hip abduction and hip adduction. Load range correction also resulted in a significant decrease in work done at velocities of $120^{\circ} \cdot \mathrm{s}^{-1}$ and above for both hip abduction and hip adduction. No subject in the present study was able to attain the velocity of $420^{\circ}$. $\mathrm{s}^{-1}$ for either hip abduction or hip adduction, however, the Biodex did still produce non-load range corrected data. This non-corrected data further highlighted the necessity for load range correction of all isokinetic data in the future. It also emphasised the importance for future investigators to trial the faster isokinetic velocities in subject practice sessions, to determine whether they can achieve the required speeds, as otherwise no load range data can be attained and peak torque and work data is fairly meaningless or largely impact artifact.

\section{Load Range, Acceleration and Deceleration}

The findings of the present study reflected past investigations in which isokinetic constant velocity movement was measured under concentric conditions. Osternig [3029] reported that knee extension load range decreased from $92 \%$ to $16 \%$ at speeds of 50 through $400^{\circ} \cdot \mathrm{s}^{-1}$. Wilk et al. [4139] also described a torque range decrease of $87 \%$ to $19 \%$ from speeds of 180 through $450^{\circ} \cdot \mathrm{s}^{-1}$ during knee extension and flexion. Also investigating the knee, Kurdak et al. [2217] found a reduction in load 
range from $94 \%$ to $4 \%$ for knee extension at speeds 30 through $390^{\circ} \cdot \mathrm{s}^{-1}$, and from $94.5 \%$ to $6.5 \%$ for knee flexion at speeds 30 through $450^{\circ} \cdot \mathrm{s}^{-1}$. Scibelli et al. [3ㅁu] demonstrated that bilateral knee extension/flexion load range decreased from $87.8 \%$ to $31.8 \%$ at speeds from 60 through $360^{\circ} \cdot \mathrm{s}^{-1}$. In addition to this, Brown et al. [8] found that load range decreased from $95.3 \%$ to $0 \%$ and from $96.3 \%$ to $21.8 \%$ during shoulder external and internal rotation, respectively, at speeds from 60 through $450^{\circ}$. $\mathrm{s}^{-1}$. The current study found that load range decreased from $92.9 \%$ to $48.2 \%$ for hip abduction, and from $93.8 \%$ to $49.3 \%$ for hip adduction, at speeds of 60 through $360^{\circ}$ $\cdot \mathrm{s}^{-1}$.

It is apparent that the results of the present study mirror the findings of the above authors $[8,21,29,34,39]$, as they all found an inverse relationship between load range and isokinetic velocity. However, direct comparisons of the results must be made with caution due to the fact that these past studies have investigated load range during unilateral knee flexion/extension [21, 29, 39], bilateral knee flexion/extension [34], and shoulder external/internal rotation [8]. No study to date has investigated the relationship between load range and velocity during concentric hip abduction and hip adduction, which makes direct comparisons difficult.

Brown and Whitehurst [3] highlighted the importance of separating the data into the three phases of acceleration, load range and deceleration. Surprisingly, some authors still fail to do this, and only consider the load range component [21]. However, Brown et al. [8] did consider the impact of acceleration and deceleration and found that both acceleration and deceleration components significantly increased with each increase in velocity. These results mirror the findings of the present study. 
However, no direct comparison of ROM (in degrees) can be made due to Brown et al. [8] studying the flexors and extensors of the knee, with a ROM of $80^{\circ}$, whereas the ROM in the present study was only $45^{\circ}$.

It is apparent that the results of the present study mirror the findings of the above authors $[8,22,30,36,41]$, as they all found an inverse relationship between load $\underline{\text { range and isokinetic velocity. Brown and Whitehurst [3] highlighted the importance of }}$ separating the data into the three phases of acceleration, load range and deceleration. Surprisingly, some authors still fail to do this, and only consider the load range component [22]. However, Brown et al. [8] did consider the impact of $\underline{\text { acceleration and deceleration and found that both components significantly increased }}$ with each increase in velocity. These results mirror the findings of the present study. However, direct comparisons of the results must be made with caution due to the fact that Brown et al. [8] studied the flexors and extensors of the knee, with a ROM of $80^{\circ}$, whereas the present study investigated the abductors and adductors of the hip with a $\underline{\mathrm{ROM} \text { of only } 45^{\circ}}$

If the results by Brown et al. [8] are converted to a percentage it can be seen that acceleration increased from $1.3 \%$ to $18.1 \%$ for knee extension, and from $1.3 \%$ to $19.1 \%$ for knee flexion, at 60 through $360^{\circ} \cdot \mathrm{s}^{-1}$. Deceleration also increased from $2.5 \% \%$ to $27.8 \%$ for knee extension, and from $2.1 \%$ to $28.0 \%$ for knee flexion, at 60 through $360^{\circ} \cdot \mathrm{s}^{-1}$. In comparison, the results of the present study found that acceleration significantly increased from $2.4 \%$ to $24.7 \%$ for hip abduction, and from $2.0 \%$ to $23.8 \%$ for hip adduction, at 60 through $360^{\circ} \cdot \mathrm{s}^{-1}$. Deceleration also significantly increased from $4.7 \%$ to $27.1 \%$ for hip abduction, and from $4.2 \%$ to 
$26.9 \%$ for hip adduction, at 60 through $360^{\circ} \cdot \mathrm{s}^{-1}$. It is clear to see there is an increase in the acceleration and deceleration components with an increase in isokinetic velocity in both studies.

In comparison to the results of Brown et al. [8] the present study showed a higher percentage of the ROM being spent in acceleration, but found a similar percentage of the ROM being spent in deceleration. Possible reasons for the differences in acceleration may be due to the different joint and musculature being tested between studies. The deceleration component was shown to be similar between studies, this may be due to using the same cushioning level as the Brown et al. [8] study, and the isokinetic dynamometer, rather than the subject themselves, was in control of this factor. However, once again direct comparisons should be made with caution due to the previously identified differences between the studies.

By quantifying ROM for the load range, acceleration and deceleration components a more complete understanding of a the isokinetic-concentric action contraction-on the isokinetic dynamometer can be achieved. The results emphasise the need for the clinician to fully understand the inverse relationship between isokinetic velocity and load range, and select the appropriate velocity accordingly. Any strength gains from training on the isokinetic dynamometer may be relative to the total amount of ROM actually sustained at the pre-selected velocity (ie, load range).

The results from the current study also emphasise the variation that exists between different joints. Even though the same general trend was indentified (load range significantly decreased while acceleration and deceleration ROM significantly 
increased with each increase in velocity), it can be seen that different joints have different levels of acceleration, load range, deceleration and maximum speed. These results further elucidate the findings that it is very important to load range correct data prior to analysis and that one cannot utilise factors from dissimilar joints. Therefore, the results from the present study should only be employed by future researchers if they are investigating the abductors and adductors of the hip.

\section{Load Range Correction for Peak Torque and Work Done}

In the present study there was a significant difference between normalised total peak torque and load range peak torque from speeds of $300^{\circ} \cdot \mathrm{s}^{-1}$ and above for both hip abduction and hip adduction. There was also a significant difference between normalised total work and load range work from speeds of $120^{\circ} \cdot \mathrm{s}^{-1}$ and above for both hip abduction and hip adduction. In agreement with these findings Kurdak et al. [221] found that the consideration of load range for peak torque and work calculations resulted in a significant decrease in the data when compared to the data presented by the isokinetic dynamometer. The authors found a significant difference between total peak torque and load range peak torque at speeds above $270^{\circ} \cdot \mathrm{s}^{-1}$ for knee extension, and above $300^{\circ} \cdot \mathrm{s}^{-1}$ for knee flexion. They also found a significant difference between total work and load range work at speeds above $90^{\circ} \cdot \mathrm{s}^{-1}$ for both knee extension and knee flexion [221]. These results highlight the importance of correcting the data for load range as it is apparent that large errors can occur if this process is not undertaken [3]. 
The normalised load range peak torque values and the normalised load range work values in the present study were lower than the results reported by Kurdak et al. [221]. However, this was expected as Kurdak et al. [221] studied the flexors and extensors of the knee joint and not the abductors and adductors of the hip joint. Unfortunately the majority of studies investigating peak torque and work of the hip abductors and hip adductors do not normalise their data to the subject's body weight $[9,19 \underline{8}, 2 \underline{3} 2,3 \underline{3} 2]$. They also do not indicate whether load range correction was completed $[9,1 \underline{9} 8, \underline{20} 19,2 \underline{3} 2,3 \underline{3} 2]$ which unfortunately makes comparisons of the data difficult. Only one study by Johnson et al. [2019] reported the data in terms of normalised peak torque values. The authors found normalised peak torque values of $0.93 \mathrm{Nm} \cdot \mathrm{Kg}^{-1}$ for the hip abductors and $1.01 \mathrm{Nm} \cdot \mathrm{Kg}^{-1}$ for the hip adductors, at an isokinetic velocity of $60^{\circ} \cdot \mathrm{s}^{-1}$ [2019]. These results are similar to the results reported in the present study which found values of $1.2 \mathrm{Nm} \cdot \mathrm{Kg}^{-1}$ and $1.0 \mathrm{Nm} \cdot \mathrm{Kg}^{-1}$ for the hip abductors and hip adductors, respectively at $60^{\circ} \cdot \mathrm{s}^{-1}$. However, no faster speeds were tested by Johnson et al. [2019] so only the comparison at $60^{\circ} \cdot \mathrm{s}^{-1}$ can be made. Johnson et al. [2019] also did not indicate if the data was reduced for load range, therefore comparisons should be made with caution as inconsistencies may be present.

Researchers should always be cautious when comparing their findings to the results of other studies as subject characteristics such as gender, age group, body weight and training history must be taken into consideration [221] ]. As well as subject characteristics, other issues such as make of isokinetic dynamometer, number of testers, experience of testers and environmental conditions should also be considered. 
The results from the present study indicate that load range corrected results are significantly different from the 'total' results produced by the isokinetic dynamometer at speeds of $300^{\circ} \cdot \mathrm{s}^{-1}$ and above for peak torque data, and $120^{\circ} \cdot \mathrm{s}^{-1}$ and above for work data, for both hip abduction and hip adduction. This trend is different to what has been found at other joints, and emphasises the fact that it is vital to load range correct data prior to analysis and that one cannot employ factors from dissimilar joints. For that reason, the results from the present study should only be utilised by future researchers if they are investigating the abductors and adductors of the hip.

\section{Mechanical vs. Physiological Deceleration}

To determine the mechanical deceleration effect of the dynamometer, a test similar to that of Brown et al. [8] was performed, to determine if a steady rate of increase in deceleration existed for each condition (mechanical vs. physiological). No significant differences were found between mechanical and physiological deceleration, however, the mechanical procedures could not be performed at the last three test velocities due to the inability of gravity to accelerate the weighted lever to speeds of 300,360 and $420^{\circ} \cdot \mathrm{s}^{-1}$ within the specified ROM. Each condition resulted in an approximate $2^{\circ}(4.4 \%)$ increase in deceleration ROM with each $60^{\circ} \cdot \mathrm{s}^{-1}$ increase in velocity. Similar results were found by Brown et al. [8] who found an approximate $4^{\circ}$ $(5.0 \%)$ increase in deceleration ROM with each $60^{\circ} \cdot \mathrm{s}^{-1}$ increase in velocity. Taylor et al. [375] found that mechanical deceleration ROM increased with a softer cushion setting, resulting in a concomitant decrease in load range. In the present study the 
cushion setting was set to zero (hard), to allow the subject the greatest availability of velocity attainment prior to deceleration [8].

\section{Joint Angle at Load Range Peak Torque}

The present study found that the joint angle at load range peak torque significantly decreased for hip abduction from $38.1^{\circ}$ to $18.3^{\circ}$, and significantly increased for hip adduction from $10.4^{\circ}$ to $34.3^{\circ}$, at 60 through $360^{\circ} \cdot \mathrm{s}^{-1}$. These results show a similar pattern to the results found by Osternig et al. [30] and Kurdak et al. [21]. These two studies showed an increase in the joint angle at load range peak torque for knee flexion, and a decrease for knee extension. However, comparisons are difficult as a different joint was investigated in contrast to the current study.

In terms of velocity prescription for the hip joint, there seems to be a lack of consensus in the literature on the most appropriate speed. The hip has very rarely $\underline{\text { been studied, and the sparse literature that is available have tested the hip in the }}$ isometric mode [33], or have tested at speeds of 60 and $90^{\circ} \cdot \mathrm{s}^{-1}$ [35]. Ferber et al. [12] found that during running at $3.65 \mathrm{~m} / \mathrm{s}(13.2 \mathrm{~km} / \mathrm{hr})$, the peak angular velocity for the hip was $103.5^{\circ} \cdot \mathrm{s}^{-1}$. Even though this speed may be far from 'explosive sporting $\underline{\text { movement' velocities, it may replicate speeds from more endurance based activities, }}$ The present study investigated a velocity spectrum from 60 to $420^{\circ} \cdot \mathrm{s}^{-1}$, but the results from Ferber et al. [12] possibly indicate that speeds close to $103.5^{\circ} \cdot \mathrm{s}^{-1}$ are $\underline{\text { most relevant and should be chosen when investigating athletes from more }}$ endurance based sports. 


\section{CONCLUSION}

In summary, the results indicate that an inverse relationship exists between load range and velocity during concentric hip abduction and hip adduction isokinetic exercise. If the velocity is not reached, the result is in absence of machine offered resistance. In addition, the results emphasise the importance of also considering the acceleration and deceleration components, as these both significantly increased with each increase in velocity, for hip abduction and hip adduction.

The results also highlight the importance of correcting the data for load range, as it is apparent that large errors can occur if this process is not undertaken. Both peak torque and work decreased following load range correction. As the isokinetic dynamometer is often used for training or rehabilitation, the results identify a need for the clinician to carefully consider velocity selection during hip abduction and hip adduction exercise. Any strength gains from isokinetic training may be proportional to the amount of time actually spent at the pre-selected velocity (ie, load range).

\section{REFERENCES}

1. Beckman SM, Buchanan TS. Ankle inversion injury and hypermobility: Effect on hip and ankle muscle electromyography onset latency. Arch Phys Med Rehabil 1995; 76: 1138-1143 
2. Brown LE, Sjostrom T, Comeau MJ, Whitehurst M, Greenwood M, Findleazy BW. Kinematics of biophysically asymmetric limbs within rate of velocity development. J Strength Cond Res 2005; 19: 298-301

3. Brown LE, Whitehurst M. Load range. In: Brown LE (ed). Isokinetics in Hhuman Pperformance. Champaign, IL: Human Kinetics Publishers, 2000: 97-121

4. Brown $L E$, Whitehurst, $M$. The effect of short term isokinetic training on force and rate of velocity development. J Strength Cond Res 2003; 17: 88-94

5. Brown LE, Whitehurst M, Bryant JR, Buchalter DN. Reliability of the Biodex System 2 isokinetic dynamometer concentric mode. Isokin Exerc Sci 1993; 3: 160163

6. Brown LE, Whitehurst M, Findleay BW. Reliability of rate of velocity development and phase measures on an isokinetic device. J Strength Cond Res 2005; 19: 189192

7. Brown LE, Whitehurst M, Findleay BW, Gilbert R, Buchalter DN. Isokinetic load range during shoulder rotation exercise in elite male junior tennis players. $\mathrm{J}$ Strength Cond Res 1995; 9: 160-164

8. Brown LE, Whitehurst M, Gilbert R, Buchalter DN. The effect of velocity and gender on load range during knee extension and flexion exercise on an isokinetic device. J Orthop Sports Phys Ther 1995; 21: 107-112

9. Claiborne TL, Timmons MK, Pincivero DM. Test-retest reliability of cardinal plane isokinetic hip torque and EMG. J Electromyogr Kinesiol 2009; 19: 345-352 


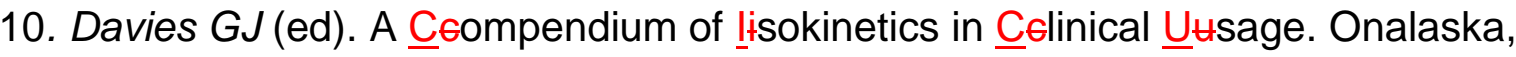
WI: S \& S Publishers, 1992: 98.

11. Emery CA, Maitland ME, Meeuwisse WH. Test-retest reliability of isokinetic hip adductor and flexor muscle strength. Clin J Sport Med 1999; 9: 79-85

\section{Ferber R, McClay-Davis I, Williams DS. Gender differences in lower extremity}

\section{mechanics during running. Clin Biomech 2003; 18: 350-357}

1ㄹz. Findleay BW, Brown LE, Whitehurst M, Keating T, Murray DP, Gardener LM. The influence of body position of load range during isokinetic knee extension/flexion. J Sports Sci Med 2006; 5: 400-406

143. Friel K, McLean N, Myers C, Caceres M. Ipsilateral hip abductor weakness after inversion ankle sprain. J Athl Train 2006; 41: 74-78

154. Gautrey CN, Watson T, Mitchell A. The effect of isokinetic testing speed on the reliability of muscle fatigue indicators during a hip abduction-adduction fatigue protocol. Int J Sports Med 2012; 33: 1-8 "in press"

1ㅁ․ Hamdoun-Kahlaoui S, Lebib S, Miri I, Ghorbel S, Koubaa S, Rahali-Khachlouf $H$, Ben Salah FZ, Dziri $C$. Isokinetic evaluation and rehabilitation of the knee in patients with patellofemoral pain syndrome. J Readapt Med 2010; 30: 3-11

176. Hammami N, Coroian FO, Julia M, Amri M, Mottet D, Herisson C, Laffont I. Isokinetic muscular strengthening after acquired cerebral damage: a literature review. Ann Phys Rehabil Med 2012; 55: 279-291

1⒎ Harriss DJ, Atkinson G. Update - Ethical Standards in Sport and Exercise Science Research. Int J Sports Med 2011; 32: 819-821 
198. Jacobs C, Uhl TL, Seeley M, Sterling W, Goodrich L. Strength and fatigability of the dominant and nondominant hip abductors. J Athl Train 2005; 40: 203-206

2019. Johnson ME, Millie ML, Martinez KM, Crombie G, Rogers MW. Age-related changes in hip abductor and adductor joint torques. Arch Phys Med Rehabil 2004; 85: 593-597

210. Kovaleski JE, Heitman RH, Trundle TL, Gilley WF. Isokinetic preload versus isokinetic knee extension resistance training. Med Sci Sports Exerc 1995; 27: 895899

221. Kurdak SS, Ozgunen K, Adas U, Zeren C, Aslangiray B, Yazici Z, Korkmaz S. Analysis of isokinetic knee extension/flexion in male elite adolescent wrestlers. $J$ Sports Sci Med 2005; 4: 489-498

23z. Laheru D, Kerr JC, McGregor AH. Assessing hip abduction and adduction strength: Can greater segmental fixation enhance reproducibility? Arch Phys Med Rehabil 2007; 88: 1147-1153

243. Makofsky H, Panicker S, Abbruzzese J, Aridas C, Camp M, Drakes J, Franco C, Sileo R. Immediate effect of grade IV inferior hip joint mobilization on hip abductor torque: a pilot study. J Manual Manipulative Ther 2007; 15: 103-111

2554. McNair PJ, Depledge J, Brettkelly M, Stanley SN. Verbal encouragement: effects on maximum effort voluntary muscle action. Br J Sports Med 1996; 30: 243-245 
265. Murray DP, Brown LE, Zinder SM, Noffal GJ, Bera SG, Garrett NM. Effects of velocity specific training on rate of velocity development, peak torque and performance. J Strength Cond Res 2007; 21: 870-874

276. Nickols-Richardson SM, Miller LE, Wooten DF, Ramp WK, Herbert WG. Concentric and eccentric isokinetic resistance training similarly increases muscular strength, fat-free soft tissue mass, and specific bone mineral measurements in young women. Osteoporos Int 2007; 18: 789-796

2ㄱ․ O'Dwyer C, Sainsbury D, O'Sullivan K. Gluteus medius muscle activation during isometric muscle contractions. J Sports Rehabil 2011; 20: 174-186

298. Osternig LR. Optimal isokinetic loads and velocities producing muscular power in human subjects. Arch Phys Med Rehabil 1975; 56: 152-155

3029. Osternig LR. Isokinetic dynamometry: implications for muscle testing and rehabilitation. In: Pandolf KB (ed). Exercise and Sport Sciences Reviews. New York, USA: Macmillan Publishing Company, 1986: 45-80

310. Osternig LR. Assessing human performance. In: Brown LE (ed). Isokinetics in Hhuman Pperformance. Champaign, IL: Human Kinetics Publishers, 2000: 77-96

321. Osternig LR, Sawhill JA, Bates BT, Hamill J. Function of limb speed on torque patterns of antagonist muscles. In: Matsui H, Kobayashi K (eds). Biomechanics. Champaign, IL: Human Kinetics Publishers, 1983: 251-257

\section{2z. Piva SR, Teixeira PE, Almeida GJ, Gil AB, DiGioia AM, Levison TJ, Fitzgerald} GK. Contribution of hip abductor strength to physical function in patients with total knee arthroplasty. Phys Ther 2011; 91: 225-233 
34⒊ Reid, $D(\mathrm{ed})$. Assessment and Rrehabilitation of $\underline{S}$ sports linjury. New York, USA: Churchill Livingstone, 1992: 43

\section{Salavati M, Moghadam M, Ebrahimi I, Arab AM. Changes in postural stability with} fatigue of lower extremity frontal and sagittal plane movers. Gait Posture 2007; 26: $\underline{214-218}$

364. Scibelli A, Brown LE, Whitehurst M, Bryant JR, Buchalter DN. Load time during bilateral isokinetic knee extension/flexion. Med Sci Sports Exerc 1993; 25: 108

375. Taylor NAS, Sanders RH, Howick El, Stanley SN. Static and dynamic assessment of the Biodex dynamometer. Eur J Appl Physiol 1991; 62: 180-188

386. Timm KE, Fyke D. The effect of test speed sequence on the concentric isokinetic performance of the knee extensor muscle group. Isokin Exerc Sci 1993; 3: $123-128$

397. Widrick JJ, Trappe SW, Costill DL, Fitts RH. Force-velocity and force-power properties of single muscle fibres from elite master runners and sedentary men. American Journal of Physiology 1996; 271: $\underline{\mathrm{C}} 676-\underline{\mathrm{C}} 683$

4038. Wilk KE, Arrigo CA, Andrews JA. Isokinetic testing of the shoulder abductors and adductors: windowed vs. Nonwindowed data collection. J Orthop Sports Phys Ther 1992; 15: 107-112

4139. Wilk KE, Romaniello WT, Soscia SM, Arrigo CA, Andrews JR. The relationship between subjective knee scores, isokinetic testing, and functional testing in the ACL reconstructed knee. J Orthop Sports Phys Ther 1994; 20: 60-70 


\section{LIST OF FIGURES}

FIGURE 1. Example of a mean velocity tracing at $60^{\circ} \cdot \mathrm{s}^{-1}$ showing acceleration (ACC), load range (LR), and deceleration (DCC) range of motion (ROM) during hip abduction.

FIGURE 2. Example of a mean velocity tracing at $360^{\circ} \cdot \mathrm{s}^{-1}$ showing acceleration (ACC), load range (LR), and deceleration (DCC) range of motion (ROM) during hip abduction.

FIGURE 3. Load range as a percentage of the total test range of motion (ROM) during hip abduction.

FIGURE 4. Load range as a percentage of the total test range of motion (ROM) during hip adduction.

FIGURE 5. Joint angle at the load range peak torque with changes in velocity.

FIGURE $\underline{5} 6$. Normalised total and load range peak torque for hip abduction with changes in velocity.

FIGURE $\underline{6}$. Normalised total and load range peak torque for hip adduction with changes in velocity. 
FIGURE 78 . Normalised total and load range work for hip abduction with changes in velocity.

FIGURE 89. Normalised total and load range work for hip adduction with changes in velocity.

\section{LIST OF TABLES}

TABLE 1. Hip abduction and hip adduction acceleration, load range and deceleration range of motion across velocities.

TABLE 2. Results of mixed factorial repeated measures analysis of variance between physiological deceleration and mechanical deceleration range of motion across velocities. 
